INTERNATIONAL DESIGN CONFERENCE - DESIGN 2018

https://doi.org/10.21278/idc.2018.0316

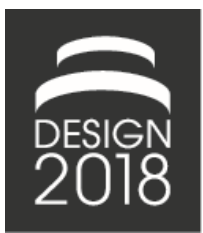

\title{
PERCEIVED QUALITY OF THE SPLIT-LINE DESIGN AND QUALITY
}

\author{
S. S. Striegel and D. Zielinski
}

\begin{abstract}
Increasing customer expectations towards high product quality and shorter product development cycles require new and standardized methods to asses a product's quality on an objective level. The split-line's design and its quality assessment correlates with the perceived quality of a product. The definitions of perceived quality are quite detailed. A lack of knowledge is the assessment process of perceived quality. Therefore, the assistance of Augmented Reality to acuire empirical data is specified. In conclusion, the overall assessment heat map is deducted and resulting standards are derived.
\end{abstract}

Keywords: perceived quality, design evaluation, tolerance representation and management, augmented reality $(A R)$, empirical studies

\section{Introduction}

Challenges within the premium automotive industry have changed over the past years. In former times technical features of premium automobiles have been one of the main reason for a customer's purchase decision. Currently various automobiles show technical similarity, i.e. similar performance in relation to maximum speed and acceleration. Therefore, an automobile's design and its quality are converting to an important purchase criterion for costumers. (Fontana et al., 1999; Falk et al., 2010; Esch, 2013; Carbon and Jakesch, 2013)

The perception of an automobile is highly subjective (Falk et al., 2010) and sometimes relates to previous knowledge of an individual (Schütz et al., 2015). A major challenge is to measure the quality or the design of products on an objective and scientific level (Carbon, 2016). Consequentially, broadscale research is conducted in the field of perceived quality of products, especially within the automotive industry. Some research approaches define perceived quality or identify quality-affecting attributes and characteristics.

Nowadays, the split-line's design and its calculation are done by a tolerance analysis. The analysis considers the calculation of a product's split-line design attitude (nominal value), its upper and its lower bounding. To support a tolerance expert's everyday business statistic tolerance simulation software, e.g. 3DCS estimate the tolerance ranges. This tolerance simulation evaluates random assembly and production tolerances along a specific tolerance chain. The result of such a simulation is a numerical value and its distribution for possible assembly results. The disadvantage of the simulation results are the lack of imagination: the emerging visual quality by a resulting tolerance range (Striegel et al., 2017). Typically, an automobile's visual split-line assessment is done with a physical prototype at a later stage of the product development process. A challenging but yet highly promising approach to speeding-up the product development process is the application of new virtual methods. Photorealistic product visualization for an early product inspection are available through ray tracing and rendering methods. Based on these visualizations a product's quality is assessable on an earlier stage of the product 
development process. To achieve an efficient visualization process and a reliable visualization results, it is necessary to identify and emulate the original assessment process of individuals.

Additionally, the analysis of various individual split-line assessments, which characteristically are highly subjective, offers the opportunity to deduce a more objective and standardized assessment approach and a scientific measurement of perceived quality.

This paper's objectives are first to identify and second to analyse the subjective assessment process of individuals in relation to an automobile's split-line design and quality. The novelty lies in the conducted empirical study. The study focuses on the subjective assessment process, the various assessment positions and the corresponding perspectives of an individual during an assessment process. To acquire the relevant data, the individual's position and its perspective are tracked frequently during the assessment process. Within the subsequent step the acquired data is prepared and analysed with the assistance of a heat map to identify distinctive assessment hot-spots. Thus, it is possible to deduce an objective assessment approach, the assessment distance, the assessment height and the corresponding assessment perspective. In addition, this process is transferable to a virtual product assessment process. In conclusion, the paper's contribution is an empirical qualitative study to identify an individual and subjective assessment process, to objectify and to standardize the results by the assistance of heat maps.

\section{Theory and literature review}

This section presents the theory and the state-of-the-art literature in the field of perceived quality and tolerance management in the context of a product quality assessment. The relation between perceived quality and the tolerance management are considered, and current research results in both research fields are described.

The focus lies on the reflection of an automobile's split-line design and quality and which characteristics an assessment approach follows.

\subsection{Perceived quality}

An automobile's visual appearance is one of its main attributes for market success (Ranscombe et al., 2012). Consequently, lots of research in the field of perceived quality is done. Stylidis et al. (2005) defines perceived quality in the automotive industry by having two attributes influencing perceived quality: Value Based Perceived Quality (VPQ) and Technical Perceived Quality (TPQ). TPQ reflects upon the technical product aspects and features, which stimulate an individual's cognitive perception in relation to the product experience. Thereby, the TPQ is divided into four sections: Visual Quality, Feel Quality, Sound Quality and Smell Quality. An automobile's split-line design and its quality relate mostly to the attribute of visual quality. VPQ considers a costumer's total product experience in relation to the product characteristics and in relation to external factors. Therefore, VPQ is calculated by including TPQ and additionally external factors (Customer Behaviour, Branding and Core Values) (Stylidis et al., 2015a, 2015b, 2016) .

Dagman et al. (2004) describe a study in which they investigate a collaboration of an automobile's visual quality and split-line design. The research's objective is to identify whether split-lines are a customer relevant product characteristic. Additionally, the study considers whether small tolerances effect a high VPQ. The conclusion of this research is a proved effect of split-lines to the VPQ of an automobile. Another study's finding is the assumption that the size of gaps and flushes are a soft factor influencing the VPQ (Dagman et al., 2004).

Research focusing on perceived quality of an automobile's split-line design is presented by Ilhan et al. (2017). Within this study perceived quality is defined as a customer's product perception and it is reached by exceeding a customer's expectations. The study's objective is to consider perceived quality and craftsmanship activities from a product's concept design to the final product. Therefore, some assumptions are made to suggest a customer's point of view. These assumptions are previously defined and evaluated by a team. By means of the resulting views, an evaluation of the Affective Usability and the Aesthetical Evaluation are done (İlhan et al., 2017; İlhan and Çelik, 2017).

The aesthetic impression of a product and the attributes influencing the product aesthetic are observed by Carbon (Carbon and Leder, 2005; Leder and Carbon, 2005; Carbon and Jakesch, 2013; Muth and Carbon, 2013). Within this research, perceived quality is defined as the reflection of a person's product 
opinion. A high level of perceived quality is achievable by a product's "liking, satisfaction or even fascination" (Carbon and Jakesch, 2013). The research focus lies on the influence of haptic and visual impression to a product's perceived quality.

Each presented research approach introduces and defines perceived quality with focus on the automotive industry. Often, a definition of perceived quality is given and a classification of different product attributes or characteristics influencing a person's cognitive system follows. Ilhan et al. (2017) presents criteria to portray a customer's view to visually evaluate a products split-line design on a functional and aesthetical level. A lack of research is evident, regarding how these criteria are deduced. In all the other presented scientific work the definition of assessment positions and perspectives are missing. To close the lack of knowledge, this paper presents an empirical qualitative study to deduce assessment positions and perspectives within an automobile's split-line assessment process.

\subsection{Tolerance management within the product development process}

Every produced product diverges from the original designed product concept due to production and assembly deviations. To handle the component deviations within the manufacturing process the product designers have to limit a component's maximum and minimum tolerances. Therefore, the tolerance management takes place within the product development process. A split-line's tolerance variation is calculated in form of a statistical distribution. A widely applied stochastically method to calculate the statistical distribution is the Monte-Carlo-Simulation. The input data are a product's 3D-CAD model, a defined tolerance chain and the tolerance ranges of each contributor in form of geometrical and assembly variations. (Bohn and Hetsch, 2013)

Figure 1 illustrates the approach to calculate a split-line's tolerance range and its corresponding statistical distribution.

Based on the resulting distribution a split-line's upper and lower limiting positions are identified. The limiting positions are defined as the minimum and maximum derivations (Ehlert et al., 2016). In relation to the customer's perceived quality and visual impression of a premium automobile a main challenge is the definition of the limiting position. On the basis of the resulting statistical tolerance distribution negative visual effects due to badly defined limits are hard to identify without having a visual feedback. The visual feedback of defined limiting positions is essential to fulfil the customer's quality expectations and to reach a high quality automobile (Striegel et al., 2017).

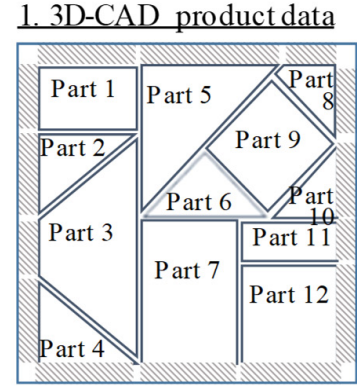

Virtual product data, showing nominal position
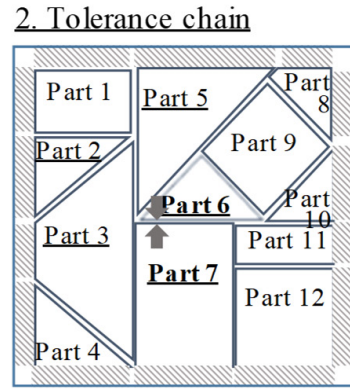

Defining two considering product parts and their tolerance chain

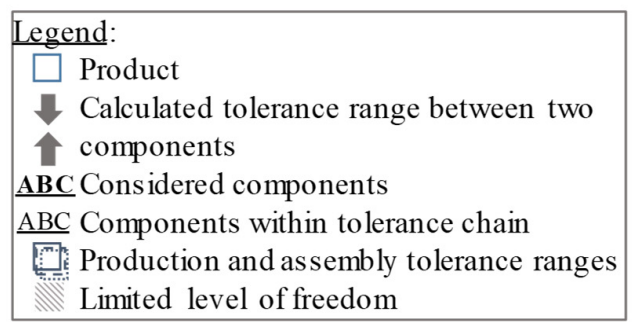

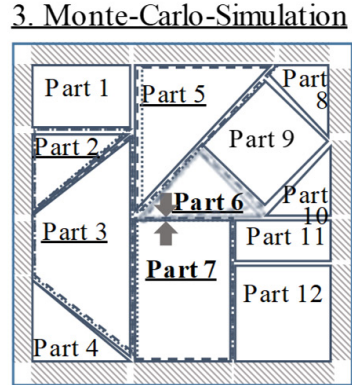

Variation of assembly and geometrical tolerances

Statistical distribution of the tolerance ranges

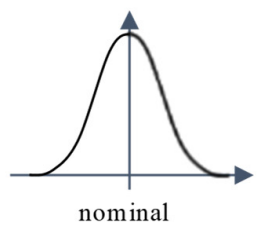

4. Upper \& lower bounds

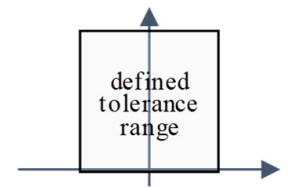

lower bound nominal bound

Internal definition of upper and lower bounds due to empirical knowledge

Resulting limiting positions Due to the overlay of the defined tolerance range and the statistical distribution

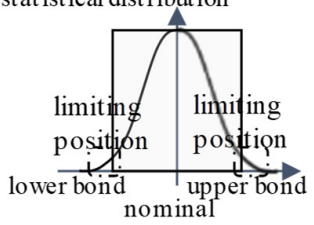

Figure 1. Calculating limiting positions by assistance of a Monte-Carlo-Simulation 
The importance to obtain a visual impression of defined limiting positions is presented by Ungemach, (2008). The developed method calculates the geometrical deformations of a component within its final assembly position in form of a FEM-simulation. In conclusion, this result is assessed due to a virtual product image.

Stoll (2012) presents a method to use virtual, imperfect prototypes to support the tolerance analysis and synthesis within the product development process. This intention is solved by two developed methods. The objective is to organize and define the product development process in a robust fashion and to reduce the number of physical prototypes due to the possibility to forecast a product's visual quality. Both presented methods investigate the use of virtual prototypes to assess a product's quality in relation to the split-line design. The lack of research lies in the unconsidered customer focused quality assessment.

\section{Research methodology}

The necessity of an empirical study, which considers an individual's assessment process of an automobile's split-line design and quality is derived from the theory and the literature review. To shed light on this research questions, a qualitative empirical study is designed and conducted. The study considers the subjects' assessment position and the corresponding perspectives and afterwards an analysis of the acquired data.

The following sections describe the study design and setting, the method to collect data and the method to analyse the collected data.

\subsection{Study design and settings}

The study has an empirical, qualitative research character. The fundamental idea is to gain knowledge and to derive standards for a split-line assessment process of individuals. Therefore, the study design is to track a subject during the quality assessment process of a real product. The focus lies on the subject's assessment approach. The study assumes to deduce objective standards for the split-line assessment by considering individual and subjective assessment approaches. This study design is illustrated in Figure 2.

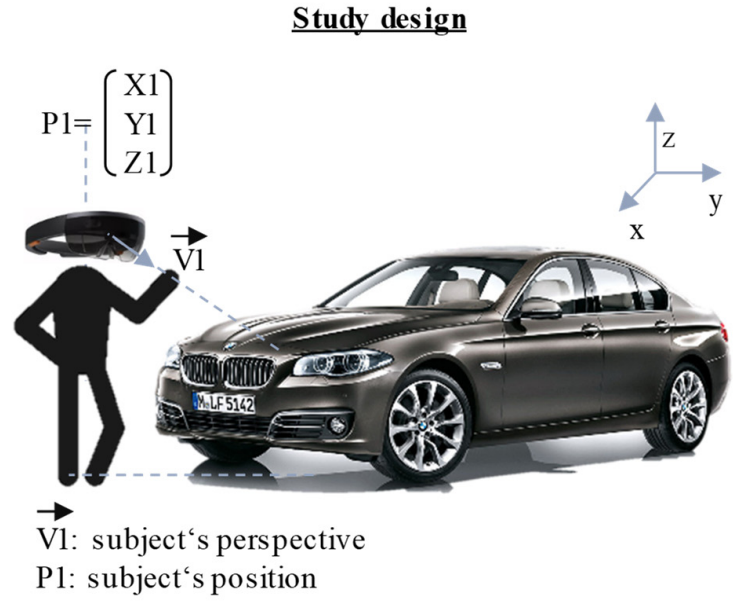

\begin{tabular}{|c|c|}
\hline \multicolumn{2}{|c|}{ Study s etting } \\
\hline OBJECT & AQUISITION \\
\hline $\begin{array}{l}\text { Total automobile's } \\
\text { exterior } \\
\rightarrow \text { split-lines }\end{array}$ & $\begin{array}{l}\text { Augmented Reality } \\
\text { device } \\
\text { Microsoft Hololens } \\
\rightarrow \text { no interaction during } \\
\text { the assessment }\end{array}$ \\
\hline CLASSES & SUBJECTS \\
\hline $\begin{array}{l}\text { Distance } \\
\text { Flatness }\end{array}$ & 14 total testing subjec \\
\hline Non-parallelism & 11 quality experts \\
\hline Symmetry & 3 laypersons \\
\hline $\begin{array}{l}\rightarrow \text { selection due to the } \\
\text { frequent appearance }\end{array}$ & $\begin{array}{l}\rightarrow \text { Comparis on of } \\
\text { various approaches }\end{array}$ \\
\hline
\end{tabular}

Figure 2. Study design and setting

Additionally, Figure 2 explains the study settings, which are following discussed. The chosen testing object is a BMW 5 series. This limousine is a classical BMW and shows similarities to variant models, e.g. the 3 series. The study-related exercise is the assessment of the total automobile's exterior in relation to the split-line design and its quality. The testing subjects are ask to detect the automobile's tolerance critical split-lines and to name the type of fault by four tolerance classes: split-line distance, flatness, parallelism and symmetry. These four tolerance classes are chosen in relation to their frequent appearance considering an automobile split-line quality. The data acquisition is supported by an augmented reality device and its use is further specified within the following section. Additionally, the 
opportunity to visualize the study's results by assistance of the chosen AR device is presented. The variety of testing subjects are 14 persons in total. Within these 14 testing subjects, 11 quality experts and 3 laypersons are asked to participate. The 11 quality experts and the 3 lay persons are chosen to contrast the expert's result with the layperson's results. There are no assessing limitations for the testing subjects due to time or duration.

The area around the testing object has about 450 square meters. This spacious room is chosen in order to ensure the testing subject ability to move freely and without restrictions to select their relevant and reasonable assessment position and perspective. Each testing subject was accompanied by an observer to document the assessment results and a timeline for uncertainties within the subsequent analysis process.

The study starts with a short briefing of the testing subjects. First, the subjects are instructed in relation to the exercise: to assess the automobile's exterior split-lines. Second, the correct use of the augmented reality device is explained.

\subsection{Data collection method}

As already outlined, the data collection is supported by an augmented reality device. In relation to the selected device two requirements are placed: a hands-free device for an unrestricted assessment and a tracking capable augmented reality device. Therefore, a head-mounted device is required and the Microsoft Hololens is chosen.

The proper use of an augmented reality is to screen additional virtual information (e.g. three-dimensional product data) on an output device that overlays the reality. Within this study the augmented reality device application are modified. To identify a subject's actual position and the corresponding perspective two tracking functions are used. First, the device current position is recorded, characterised by a three-dimensional spot. Second, the perspective's normal is tracked in form of a vector. Both tracking functions (spot tracking and vector tracing) are setup with a frequency of six times per second and during the total assessment process. The three-dimensional spot and the vector are illustrated in Figure 3.

Additionally, the virtual product data is implemented to the augmented reality device. The virtual product information is required in three cases:

1. Verifying a consistent tracking

2. Referring the acquired data to the real product

3. Analysing the collected data in relation to the real product dimensions

The virtual product data overlay makes the assessment of the real product difficult. Therefore, the virtual data is invisible for the participants during the study.

Figure 3 sketches out the virtual data overlay and the data collection method during the assessment process.

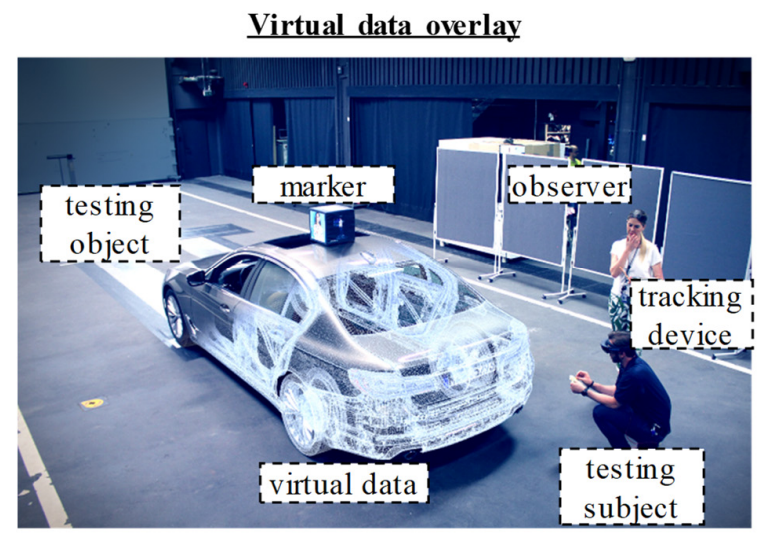

Data collection method

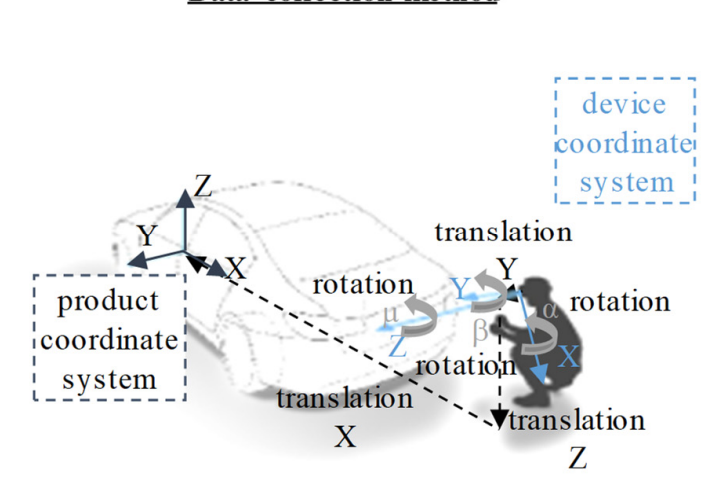

Figure 3. Virtual data overlay and data collection method

The data collection method is based on the position and perspective tracking function. The tracking device screens the surrounding area and is able to notice each location. By using this screening method 
the current position can be tracked in the selected frequency. The position tracking process is similar to the perspective tracking process. The difference between both tracking processes is the output. The location tracking results in a three-dimensional spot. The result of the perspective tracking is a vector. The major challenge of the data collection is a coordinate transformation of the device coordinate system to the product coordinate system. The solution is a translation and rotation motion of the device coordinate system. Therefore, the rotation angles $(\alpha, \beta, \mu)$ and the translation distance $(X, Y, Z)$ are required. Based on this information a transformation matrix is deductible. By having the resulting matrix, each tracked spot and vector can be translated to the product coordinate system. Figure 3 sketches the coordinate transformation of the tracking device to the product coordinate system.

\subsection{Data analysis method}

This section focuses on the data analysis method. To derive the assessment standards the data analysis method considers four topics: areas of interest, distance, height and view angle. By means of these four topics it is possible to deduce a method in relation to the relevant split-line assessment position and the corresponding perspective.

To investigate the four topics the collected data is processed and a heat map for each individual inspection is deduced. The data processing approach is presented in Figure 4.

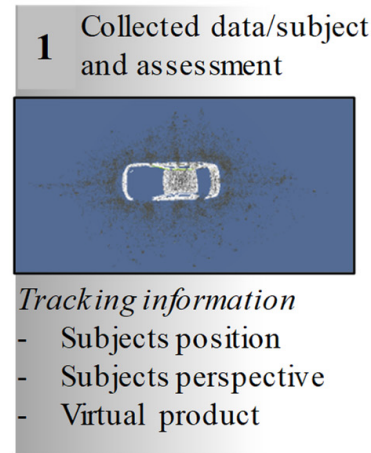

Subjects perspective
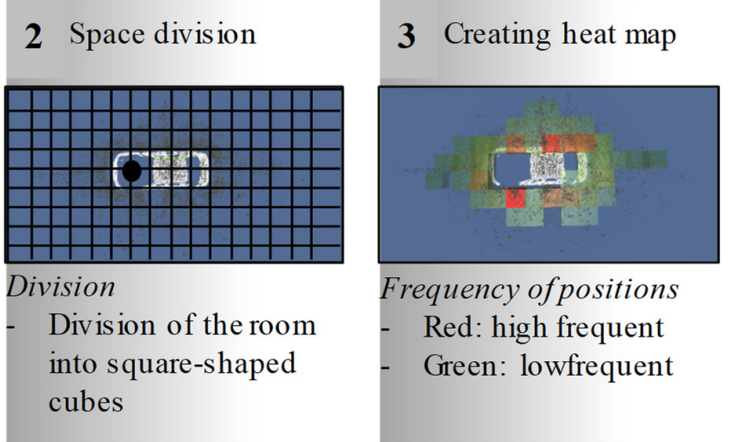

Frequency of positions

Red: high frequent

Green: lowfrequent

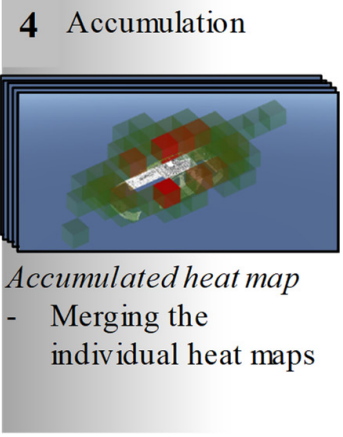

Figure 4. Data analysis approach

The first step presents the collected data in form of grey spots of the tracked positions. To create the heat map the starting point is the coordinate origin of the assessed automobile. Afterwards, the surrounding is divided into square-shaped cubes. The cube colour is defined by a subject's activity due to their frequency of stay within this cube. The colour scale is derived as follows:

1. Counting the measured values per cube

2. Identifying the maximum and the minimum amount of measured values

3. Calculating the colour scale per cube: cube colour $=$ number of measured values/maximum amount

4. Colour scale:

cube colour $=1 \rightarrow$ red

$1<$ cube colour $<0 \rightarrow$ green

cube colour $=0 \rightarrow$ blue

The third step results in a heat map of an individual assessment process. The overall heat map is an accumulation of the several various heat maps. Therefore, the individual spots and vectors are added to the spots and the vectors of further heat maps. This way of data processing and the resulting heat map based on a subject's position and perspective tracking enables investigating the four topics: areas of interest, distance, height and angle of view. The following sections specify these four topics and give a short definition for each topic.

\section{Areas of interest}

By assistance of the heat map's cube colour the areas of interest can be identified. The red cube colour describes the areas with the highest frequency of stay within the assessment process. Whereas the colour 
blue or light green classifies areas with less frequency of stay. The study's resulting overall heat map and the scale of a subject's activity due to their frequency of stay are presented in Figure 5.

\section{Distance}

The assessment distance is deducted by observing the square's size. By getting the information in relation to the relevant $\mathrm{X}$ - and $\mathrm{Y}$-assessment direction the bird's-eye perspective of the resulting heat map is useful. The study cube dimensions are 0,8 by 0,8 meters. The assessment distance behaves as following. One square: 0 meter up to 0,8 meter, two squares: 0,8 meter to 1,6 meter. The resulting heat map in relation to the assessment distance is illustrated in Figure 5.

\section{Height}

The analysis of the assessment height is derived similarly as the analysis of the assessment distance. By observing a heat map square and its size, the assessment height can be investigated. The geometrical definition of the squares has 0,8 meters in each direction. The reasonable assessment height is readable by the support of the resulting heat map. Therefore, the front, side or rear view of the assessed object is reliable. Figure 5 presents the resulting heat maps with an exemplary side view of the automobile.

\section{Angle of view}

The consideration of the angle of view requires the previous findings due to the tracked positions. The perspective vector of each tracked position by the augmented reality device is identified in conclusion of the study. Thus, not every tracked and recorded perspective during the assessment process is useful. Perspectives, which affect or even pass by the virtual exterior of the automobile are filtered out and are not further considered. The resulting filtered perspectives are further considered. Starting with a position with a high frequency of stay every corresponding perspective vector needs to be accumulated and a perspective funnel results. By assistance of this funnel and the virtual product data the individual's current inspected area of the automobile is reconstructable. Due to the derived funnel and the resulting assessment section a virtual assessment position, e.g. a rendering camera position is deductible. This approach to detect the angle of view based on the current position is presented in Figure 5.

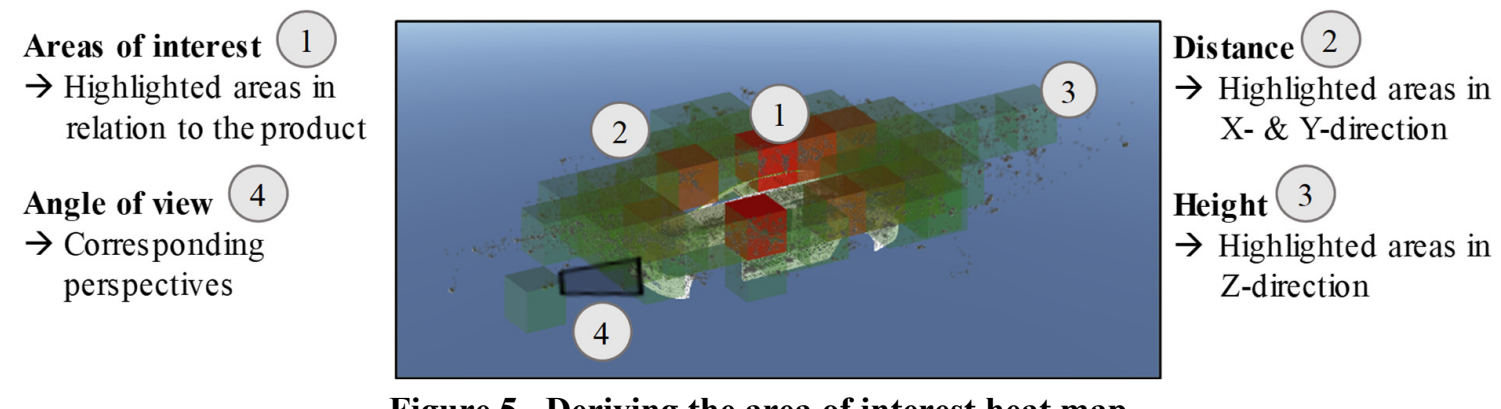

Figure 5. Deriving the area of interest heat map

\section{Results}

In conclusion of the previous sections the state-of-the-art research in relation to perceived quality and tolerance management and the presented research methodology used in the current study, this section considers the obtained results. The following presented results are specified by using the four previous introduced topics.

\section{Areas of interest}

Three main areas of interest can be identified by observing the resulting overall heat map and the cube colour. The study's overall heat map is presented in Figure 6 and includes the accumulated heat map of every individual participating the assessment study. 


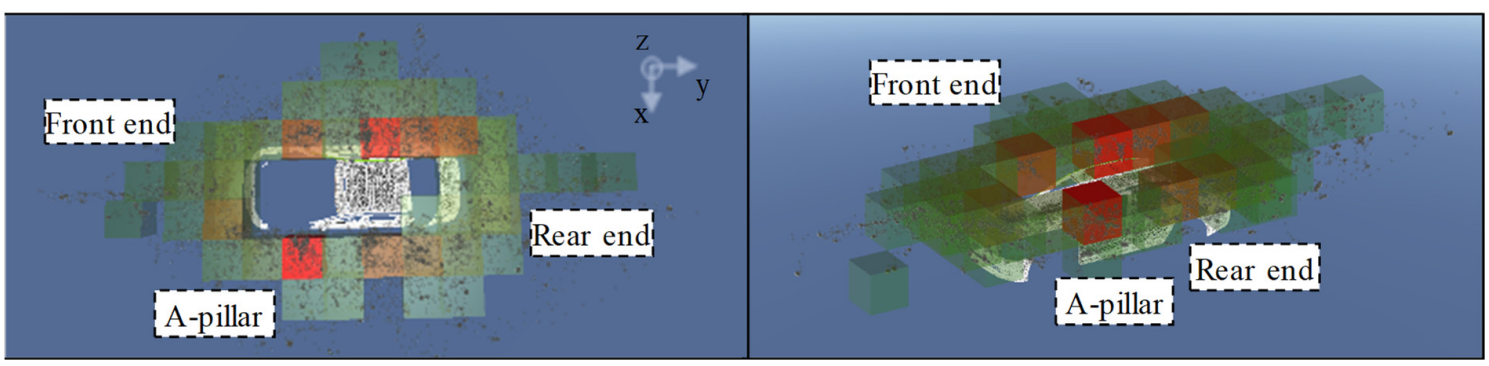

Figure 6. Three main areas of interest

First, a symmetrical automobile assessment process is significant. In particular, the red marked areas are consistent at the driver's and co-driver's side. The identified areas of interest are the A-pillar, the rear and the front end. An additional interesting finding is the assessment's starting point: each participating subject starts its assessment process with the entry of driver's side. This recognition is supported by the additionally documented information in relation to the split-line's four type of faults. Going into detail the first red coloured hot spot at the A-pillar focus the triangle including the chassis, side mirror, the front flap and the side panel. Due to the many involved product components the tolerance management is highly complex and affects the visual product appearance. The detailed observation of the second area, the front-end area, detects the area around the front flap, the front-end headlight and the design element kidney grille. In comparison to the A-pillar area, the number of components is less but the involved components are mostly design parts, e.g. the BMW typical kidney or the vehiclespecifically headlights. The third hot spot area contains the rear entry, the rear chassis, the rear flap and the rear headlights. At the co-driver's rear entry, the squares are darker red compared to the driver's side. This higher activity can be explained by the fuel filler flap, which is placed at this side of the automobile. The fuel filler flap requires a higher assessment effort. Thus, the subject's frequency of stay is higher at this side of the automobile.

The three areas of interest shed light on the question, which automobile's sections are with a higher quality assessment focus. The following consideration of the distance and height focuses on the threedimensional orientation of the assessment position of individuals.

\section{Distance}

The second issue considers the assessment distance of the individual assessment process. Therefore, the resulting heat map and the virtual product data are presented in Figure 7a.

The main assessment distance is identifiable by considering the bird's-eye view. During the assessment process the main distance is 0,8 meters. The presented square's size is 0,8 meters in each $\mathrm{x}, \mathrm{y}, \mathrm{z}$ direction. Besides the main distance receiving form the bird's-eye view, the front and rear end sides are viewed with a greater distance up to 3,2 meters at the front and rear end. Considering the heat map with the rear end view the distance to assess the automobile's driver and co-driver side is achieved.

\section{Height}

The derivation of the height within the split-line's assessment process is possible by means of the identical heat map the distance analysis is done. Considering Figure $7 \mathrm{~b}$ and $7 \mathrm{c}$ the heat maps side view and rear end view is meaningful.

The observing height is readable by the coloured squares. Noticeable is the main assessment height by having two squares which corresponds with a person's eye height: 1,6 meters. Considering the rear view another additional and reasonable height is remarkable: one heat map square, which means 0,8 meters for the assessment height.

Summarizing, the main height results with the eye height by having 1,6 meters.

\section{Angle of view}

The observation of the angle of view is based on the prior results which deal with the assessment position. The angle of view is deduced by obtaining a subject's current position due to the assessment distance and height. Figure $7 \mathrm{~d}$ illustrates the different viewing vectors obtaining with the augmented 
reality device's tracking. In combination to each assessment position the corresponding perspective is presented. The result is a confusing heat map with many perspective vectors. To increase the transparency the correlating vectors are accumulated to a funnel. The separated angle of view is presented in Figure 8.

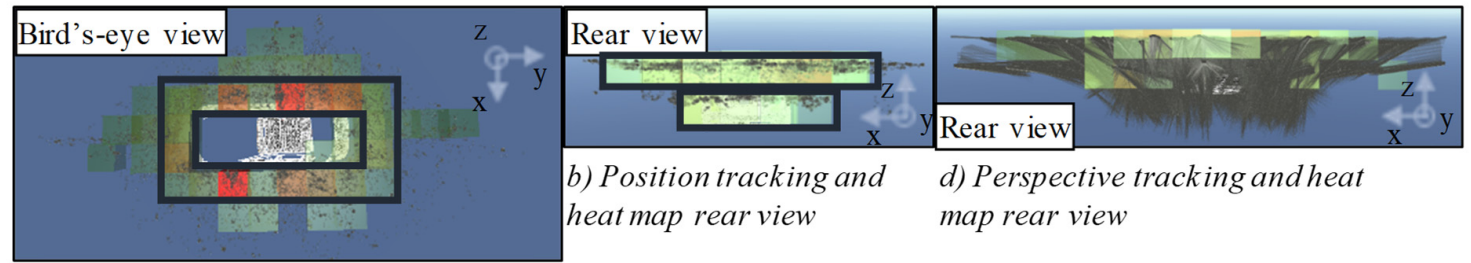

a) Position tracking and heat map bird:-

eye view

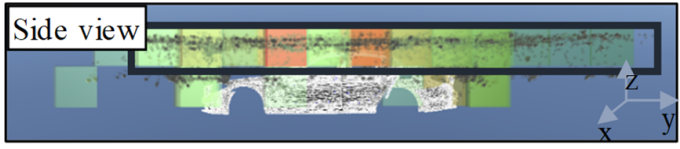

c) Position tracking and heat map side view

\section{Figure 7. Results in form of the heat maps}

The study's main finding is the few assessment positions and the large viewing angle within the assessment process. Especially, the assessment positions in the front and rear by having a distance up to 3,2 meters, the angle of view is the whole automobile. Regarding the a-pillar position within the assessment process similar conditions are detectable. The angle of view is large and considers almost the whole side of the car. The resulting assessing positions and their corresponding perspectives are summarized in Table 1.

In conclusion to the empirical study the question how individuals assess an automobile's split line design and quality, the answer is: individuals assess an automobile's split line design and quality by few positions but with a large angle of view.

Table 1. Resulting assessment positions and perspectives

\begin{tabular}{|l|c|c|r|}
\hline Area of interest & Distance & Height & Angle of view \\
\hline front end & 0,8 meter $-3,2$ meter & 0,8 meter $-1,6$ meter & wide-angle: total car \\
\hline a-pillar & up to 0,8 meter & 1,6 meter & small angle: details \\
\hline rear end & 0,8 meter $-3,2$ meter & 0,8 meter $-1,6$ meter & wide-angle: total car \\
\hline
\end{tabular}

\section{Discussion}

The necessity of the empirical study is derived by the state-of-the-art review and the recent research. Perceived quality within the premium automobile industry is discussed and investigated by many researchers. Definitions, requirements and relations between the product perceptions are explored. The lack of knowledge lies within the consideration of an actual and subjective assessment approach. Therefore, the empirical study considers an automobile's split line design and quality assessment process of individuals.

The results are investigated due to four issues: areas of interest, assessment distance, height and corresponding perspectives. The four issues indicate which sections of the assessed automobile are within higher quality pressure and how they were assessed. This gained transparency offers the possibility of an assessment process with objective standards independent of the assessed automobile. These standards are universally applicable in alternating assessment situations. Each corresponding perspective focuses on the split lines, thus the perspective while considering another automobile is eventually adaptable with adjustments as well. This hypothesis requires further comparison between the overall heat maps of different automobile models. E.g. the overall heat map, including the corresponding perspectives, of a sedan model and a SUV model. Thereby assessment equalities and the differences 
between various automobiles can be observed. Also, a comparison of several automobile companies and their overall heat map of one automobile class is considerable.

An opportunity to visualize the results is to use the augmented reality device in its common use. Therefore the resulting heat map including the accumulated positions and perspectives is presented as a virtual feature and overlay the reality. This visualisation is an immersive technique to present the results to various persons. Figure 8 presents the virtual overlay of the resulting heat map and a resulting perspective by the assistance of an AR device.

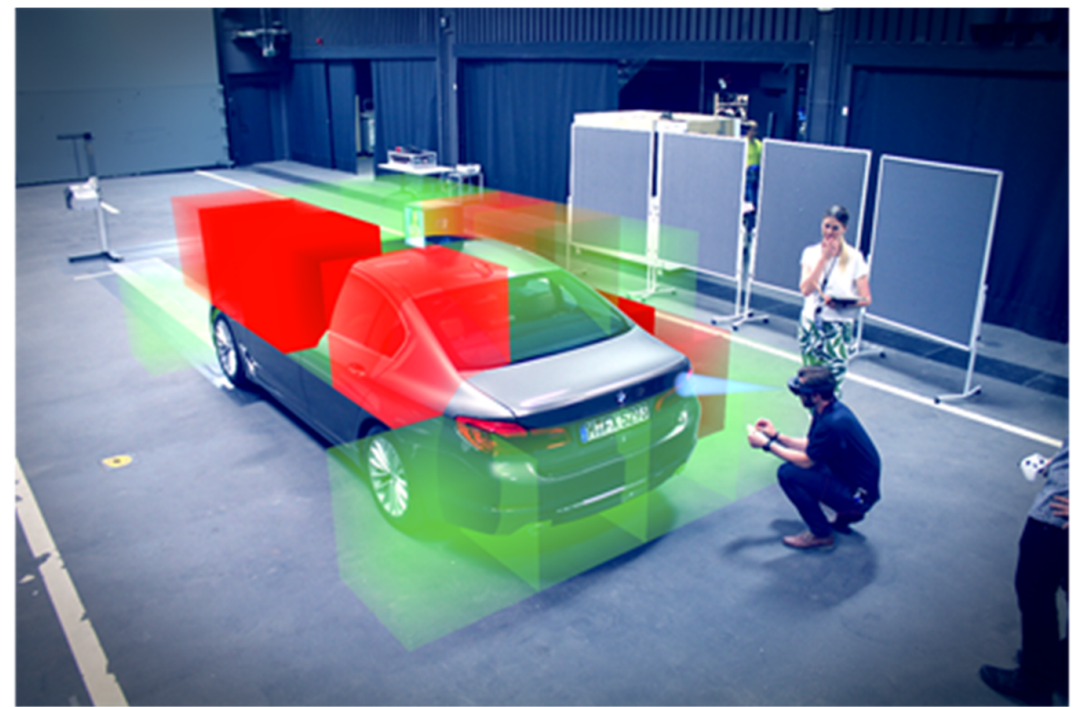

Figure 8. Opportunity to report the study results by the assistance of an AR device

An additional application is the transfer of the tracked positions and the corresponding perspective to a virtual assessment approach within the product development process. For example, the chance to define camera positions for a virtual rendering or raytracing software by use of the study's deduced position and the corresponding perspective funnel. In this way, a justifiable selection of camera positions and perspectives in relation to a considered split-line assessment is determining by assistance of the study results.

At this point it is transparent which positions and corresponding perspectives are necessary for an assessment, but the product's displayed tolerance situation needs further research. The product assessment is placed in an early stage of the product development process and thus the type and frequency of tolerance failure is not available. Therefore, a realistic tolerance situation and the components variation has to be forecasted. Additionally, a visual tolerance limit equal to the functional limiting position needs to be identified.

Alternative to the perspective tracking, a further and more precise eye tracking can be investigated. The results of an eye tracking can then be used to further validate the perspective tracking.

\section{Conclusion}

The introduced study deals with the research question of how individuals assess an automobile's splitline design and quality. The necessity is derived by the state-of-the-art and recent research results within the topics of perceived quality and tolerance management. In conclusion to the presented definitions and relations between perceived quality and the tolerance management within the automobile industry, the study's design and scope is presented. The study is supported by an augmented reality device. The device tracks an individual's current position and its corresponding perspective. Afterwards, this information is analysed in relation to the four considered issues: area of interest, distance, height and angle of view. By analysing these four issues an individual's assessment position and perspective can be reconstructed. In doing so, three main assessment positions and the corresponding perspectives can be identified. The areas of interest are at the A-pillar, the front and the rear end and with the assessment 
distance up to 8,0 meters and the assessment height of eye level. The perspective angle at each assessment position is large. In short, individuals assess an automobile's split-line design and quality with few assessment positions but with a large perspective angle.

Further research issues might have to deal with the transferability of the results to variant automobile models and the identification of a visual tolerance limit. Additionally, the transferability of the results to a virtual assessment method needs to be evaluated. Besides, the limits of a virtual assessment method need to be detected in relation to the product perception and the virtual restrictions.

This paper's study of automobile split-line quality assessment initiate further critical research questions. What areas of an automobile need to be assessed regarding split-lines? And how can visual limits in relation to the split-line design and quality be detected. Based on the result of these additional research questions a comprehensive and global method to assess an automobile's split line quality using a rendering or raytracing method can be evolved.

\section{Acknowledgment}

The authors wish to thank Björn Schwerdtfeger, who has supported the study conduct. Additionally, the authors thank Sascha Ballardt, Peter Baintner, Dominik Zeschke and all trial participants.

\section{References}

Bohn, M. and Hetsch, K. (2013), Toleranzmanagement im Automobilbau, Carl Hanser Verlag, München. https://doi.org/10.3139/9783446435445

Carbon, C.-C. (2016), "Creating a Framework for Holistic Assessment of Aesthetics: A Response to Nilsson and Axelsson (2015) on Attributes of Aesthetic Quality of Textile Quality", Perceptual and motor skills, Vol. 122 No. 1, pp. 96-100. https://doi.org/10.1177/0031512516628366

Carbon, C.-C. and Jakesch, M. (2013), "A Model for Haptic Aesthetic Processing and Its Implications for Design”, Proceedings of the IEEE, Vol. 101 No. 9, pp. 2123-2133. https://doi.org/10.1109/JPROC.2012.2219831

Carbon, C.-C. and Leder, H. (2005), "The Repeated Evaluation Technique (RET). A method to capture dynamic effects of innovativeness and attractiveness", Applied Cognitive Psychology, Vol. 19 No. 5, pp. 587-601. https://doi.org/10.1002/acp.1098

Carbon, C.-C. (2016), "Beyond the predominance of the visual empire: a functional model on haptics \& more", Electronic Imaging, Human Vision and Electronic Imaging 2016, pp. 1-2. https://doi.org/10.2352/ISSN.24701173.2016.16.HVEI-098

Dagman, A., Wickman, C. and Söderberg, R. (2004), “A Study of Customers' and the Automotive Industry's Attitude Regarding Visual Quality Appearance of Split-Lines", 4th International Conference on Advanced Engineering Design AED'2004, At Glasgow, Scotland, UK.

Ehlert, M., Hofmann, R., Stockinger, A. and Wartzack, S. (2016), “On the Evolvement of Limiting Positions as a Result of the Interaction of Tolerances and Compliant Systems", Procedia CIRP, Vol. 43, pp. 76-81. https://doi.org/10.1016/j.procir.2016.02.150

Esch, F.-R. (2013), Strategie und Technik des Automobilmarketing, Springer Gabler, Wiesbaden. https://doi.org/10.1007/978-3-8349-3831-2

Falk, B., Quattelbaum, B. and Schmitt, R. (2010), "Product Quality from the Customers' Perspective - Systematic Elicitation and Deployment of Perceived Quality Information", Proceedings of the 6th CIRP-Sponsored International Conference on Digital Enterprise Technology, Advances in Intelligent and Soft Computing, Vol. 66, Springer, Berlin, Heidelberg, pp. 211-222. https://doi.org/10.1007/978-3-642-10430-5 16.

Fontana, M., Giannini, F. and Meirana, M. (1999), “A Free Form Feature Taxonomy”, Computer Graphics Forum, Vol. 18 No. 3, pp. 107-118. https://doi.org/10.1111/1467-8659.00332

İlhan, E.Ü. and Çelik, H. (2017), "Perceived Quality and Craftsmanship-Common Terminology Proposal for Automotive World with Guidance of Kansei Engineering”, Proceedings of the AHFE 2016 International Conference on Affective and Pleasurable Design, July 27-31, 2016, Walt Disney World, Florida, USA, Advances in intelligent systems and computing, Vol. 483, Springer, Cham. https://doi.org/10.1007/978-3-31941661-8 38

İlhan, E.Ü., Ünal, C.U. and Altınok, Y.E. (2017), "Perceived Quality and Craftsmanship: An Engineering Approach to Trunk Lid Cutline: Advances in affective and pleasurable design", Proceedings of the AHFE 2016 International Conference on Affective and Pleasurable Design, July 27-31, 2016, Walt Disney World, Florida, USA, Advances in intelligent systems and computing, Vol. 483, Springer, Cham.

Leder, H. and Carbon, C.-C. (2005), "Dimensions in appreciation of car interior design", Applied Cognitive Psychology, Vol. 19 No. 5, pp. 603-618. https://doi.org/10.1002/acp.1088 
Muth, C. and Carbon, C.-C. (2013), "The aesthetic aha: on the pleasure of having insights into Gestalt", Acta psychologica, Vol. 144 No. 1, pp. 25-30. https://doi.org/10.1016/j.actpsy.2013.05.001

Ranscombe, C., Hicks, B., Mullineux, G. and Singh, B. (2012), "Visually decomposing vehicle images. Exploring the influence of different aesthetic features on consumer perception of brand”, Design Studies, Vol. 33 No. 4 , pp. 319-341. https://doi.org/10.1016/j.destud.2011.06.006

Schütz, A., Brand, M. and Selg, H. (2015), Psychologie: Eine Einführung in ihre Grundlagen und Anwendungsfelder, 5th ed., Kohlhammer Verlag, Stuttgart.

Stoll, T. (2012), Einsatzmöglichkeiten von virtuellen, nichtidealen Prototypen in der Toleranzsynthese und analyse, PhD thesis, Universität Erlangen-Nürnberg.

Striegel, S., Schleich, B., Zielinski, D. and Wartzack, S. (2017), “Automotive Premium Quality Improvement by High-End-Visualization", Proceedings of the ASME 2017 International Mechanical Engineering Congress and Exposition. https://doi.org/10.1115/IMECE2017-71218

Stylidis, K., Burnap, A., Rossi, M., Wickman, C., Söderberg, R. and Papalambros, P.Y. (2016), “A Preliminary Study of Trends in Perceived Quality Design Attributes in the Automotive Luxury Market Segment", Proceedings of the DESIGN 2016 / 14th International Design Conference, Dubrovnik, Croatia, May 16 - 19, 2016, The Design Society, Glasgow, pp. 2189-2196.

Stylidis, K., Landahl, J., Wickman, C., Johannesson, H. and Söderberg, R. (2015a), “Structuring Perceived Quality Attributes for use in the Design Process", Proceedings of the 20th International Conference on Engineering Design (ICED 15), Milan, Italy, The Design Society, Glasgow, pp. 33-42.

Stylidis, K., Wickman, C. and Söderberg, R. (2015b), "Defining Perceived Quality in the Automotive Industry. An Engineering Approach", Procedia CIRP, Vol. 36, pp. 165-170. https://doi.org/10.1016/j.procir.2015.01.076

Ungemach, G. (2008), "Visualisation of Component Deformation for Virtual Quality Assessment", ATZ, Vol. 2008 No. 110, pp. 76-79. https://doi.org/10.1007/BF03225019

Serena Simone Striegel, Master of Science

BMW Group, Total Vehicle Validation (Production)

Knorrstraße 143, 80788 Munich, Germany

Email: serena.striegel@bmw.de 